\title{
Composition, diversity and short-term temporal fluctuations of zooplankton communities in fish culture ponds (Pindamonhangaba), SP
}

\author{
Negreiros, NF. ${ }^{\text {a* }}$ Rojas, NE., ${ }^{\mathrm{b}}$ Rocha, $O .^{\mathrm{c}}$ and Santos Wisniewski, MJ. ${ }^{\mathrm{d}}$ \\ aPrograma de Pós-Graduação em Ecologia e Recursos Naturais, Universidade Federal de São Carlos - UFSCar, \\ CP 676, CEP 13565-905, São Carlos, SP, Brazil \\ 'Instituto de Pesca, \\ Rod. Washington Luís, Km 445, CP 1052, CEP 15025-970, São José do Rio Preto, SP, Brazil \\ 'Departamento de Ecologia e Biologia Evolutiva - DEBE, Universidade Federal de São Carlos - UFSCar, \\ CP 676, CEP 13565-905, São Carlos, SP, Brazil \\ ${ }^{\mathrm{d}}$ Universidade Federal de Alfenas - UNIFAL, \\ Rua Gabriel Monteiro da Silva, 714, CEP 37130-000, Alfenas, MG, Brazil \\ *e-mail: natalia_felix@yahoo.com.br
}

Received October 8, 2007 - Accepted May 12, 2008 - Distributed August 31, 2009

(With 7 figures)

\begin{abstract}
The present study aimed at evaluating the composition, diversity and short-term temporal fluctuations of zooplankton communities in fish ponds. The study was carried out in two fish ponds, with $180 \mathrm{~m}^{2}$ of water surface $(6 \times 30 \mathrm{~m})$ each, located in the Aquiculture Centre of the Pindamonhangaba Fisheries Institute - São Paulo. The study was developed over eight weeks, from February 16 to April 6, 1998. The physical and chemical conditions of the water in the fish ponds were adequate for zooplankton development. The zooplanktonic community was characterised by high richness of species and a greater diversity was observed in the first fish pond, with a superior density of Rotifera. Temporal changes in zooplankton composition occurred in both ponds with Cladocera appearing in abundance later, in the fourth week, whereas copepods and rotifers were well represented since the beginning. Many species found are typical of fish ponds and are considered to constitute an excellent food source, showing high nutritional value for fish larvae, a good example being individuals from the Rotifera group and the micro-crustacean species Moina minuta and Thermocyclops decipiens.
\end{abstract}

Keywords: fish pond, zooplankton, diversity, temporal fluctuations.

\section{Composição, diversidade e flutuações temporais de curto prazo da comunidade zooplanctônica de viveiros de piscicultura (Pindamonhangaba), SP}

\begin{abstract}
Resumo
O presente estudo visou avaliar a composição, a diversidade e a flutuação do zooplâncton em dois viveiros escavados na terra com $180 \mathrm{~m}^{2}$ de espelho d'água $(6 \times 30 \mathrm{~m})$ cada um, no Núcleo de Aquicultura do Instituto de Pesca de Pindamonhangaba-SP. O estudo foi realizado durante oito semanas, no período de 16 de fevereiro a 6 de abril de 1998. As condições observadas, em relação às características físicas e químicas da água dos viveiros, foram adequadas ao desenvolvimento dos organismos zooplanctônicos. A comunidade zooplanctônica foi caracterizada por elevada riqueza de espécies, com maiores densidades de organismos pertencentes ao grupo Rotifera. Mudanças temporais na composição do zooplâncton ocorreram em ambos os tanques, com Cladocera aparecendo em maior abundância mais tarde, na quarta semana, enquanto que copepodos e rotíferos foram bem representados desde o início. Muitas espécies encontradas são típicas de viveiros de piscicultura e constituem excelente fonte alimentar, apresentando alto valor nutritivo para larvas de peixes, como por exemplo os indivíduos do grupo Rotifera e as espécies Moina minuta e Thermocyclops decipiens.
\end{abstract}

Palavras-chave: viveiros, zooplâncton, diversidade, flutuações temporais. 


\section{Introduction}

In recent years, freshwater aquaculture has undergone many advances due to efforts made to increase fish production (Sipaúba-Tavares et al., 1994). Shallow artificial reservoirs, such as fish-ponds, are extremely dynamic, due to wind action, precipitation and continuous water flow, presenting planktonic communities with peculiar characteristics due to the short residence time and the wide fluctuations of environmental characteristics (Rietzler and Rocha, 2000).

In fish cultures, water quality and the success in its management depend on a strong knowledge of the structure and functioning of the ponds, particularly with regard to the water's physical and chemical variables and to the biological communities (Sipaúba-Tavares et al., 1994).

The fresh water zooplankton is constituted mainly by microcrustaceans belonging to the Cladocera and Copepoda groups as well as by the Rotifera, in addition to some individuals from minor groups. In a eutrophic environment, the zooplankton community is usually dominated by the micro-zooplankton (Rotifera, Protozoa and copepod nauplii) (Sampaio et al., 2002). In fish culture activities, plankton production is a factor of great importance since it constitutes the most adequate food for the fish in their youngest phase, especially the rotifers and the cladocerans; the presence of these organisms in the environment may lead to better larval development (Feiden and Hayashi, 2005).

The diversity of zooplankton species in fish ponds is usually high, especially regarding rotifers, and many species can be used as water quality indicators (Macedo and Sipaúba-Tavares, 2005).

As in natural ecosystems, the zooplankton community of such ponds is also influenced by environmental variables, being dependent especially on the $\mathrm{pH}$, alkalinity and temperature, as well as dissolved oxygen, nitrogen, phosphorus and ammonia concentrations (SipaúbaTavares and Moreno, 1994).

The present study attempted to evaluate the relations between zooplankton composition, diversity and shortterm fluctuations of abundance, and environmental factors in two fish ponds at the Aquaculture Centre of the Pindamonhangaba Fisheries Institute - São Paulo.

\section{Material and Methods}

The study was carried out in two fish ponds, dug in the ground, of $180 \mathrm{~m}^{2}$ of water surface $(6 \times 30 \mathrm{~m})$ each, which were stocked with tilapia larvae (Oreochromis niloticus) with initial densities of about 128 to 131 larvae per $\mathrm{m}^{2}$. During 50 days data regarding water temperature, water transparency (Secchi disk), $\mathrm{pH}$ and electrical conductivity were recorded weekly. The depth of the euphotic zone was calculated multiplying the value of the Secchi disk reading by the empirical coefficient of extinction, equal to 2.7 (Margalef, 1983). Each week, the concentrations of ammonia, nitrate, nitrite and dissolved and total phosphorus in the pond waters were determined. Diurnal variations of dissolved oxygen concentrations (D.O.) were analysed (measurements every 6 hours) at weekly intervals. Suspended solids concentrations were also measured once a week. These analyses followed the APHA (1998) methodology. Chlorophyll $a$ concentration was determined weekly, using the methodology described in Golterman and Clymo (1969), with the modifications proposed by Wetzel and Likens (1991).

Zooplankton was collected weekly by horizontal net hauls in all the ponds' extension $(30 \mathrm{~m})$, using a plankton net with a mesh size of $60 \mu \mathrm{m}$. The volume filtered was calculated using the equation for calculating the volume of a cylinder $\left(\omega r^{2} h\right)$ being $r$ the value of half of the diameter of the net mouth. The organisms were preserved in $4 \%$ formaldehyde. The qualitative and quantitative analyses were done under a stereoscopic microscope (50x) and an optical microscope $(2,000 \times)$. For taxonomic identifications, a specialised bibliography was utilized (Edmondson, 1959; Koste, 1978; Reid, 1985; Segers, 1995; Elmoor-Loureiro, 1997; Silva and MatsumuraTundisi, 2005). Counting of the organisms belonging to the Cladocera and Copepoda groups was performed in squared acrylic plaques, using sub-samples, or the whole sample for rare organisms. For the Rotifera, sub-samples of $1 \mathrm{~mL}$ in a Sedgewick-Rafter chamber were utilised.

Diversity Shannon-Wiener index (in base 10) was calculated (Magurran, 1998). The constancy index was calculated considering: constant the species that occurred in more than $50 \%$ of the samples, accessory species that occurred in more than $25 \%$ and rare or accidental the species that occurred in less than $25 \%$ the samples (Dajoz, 1983).

\section{Results}

In Tables 1 and 2, the values relative to the parameters of the physical and chemical variables are shown. Figures 1 and 2 show the diurnal variations in dissolved

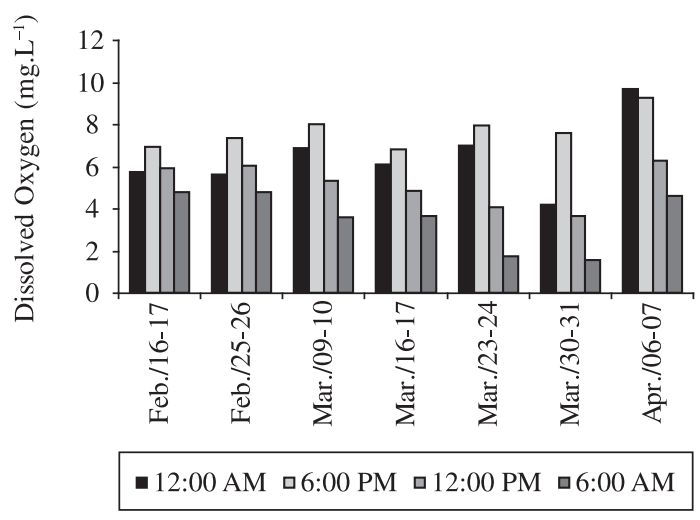

Figure 1. Diurnal variation of dissolved oxygen in pond 1, in Pindamonhangaba Aquaculture Station, from February to April, 1998. 


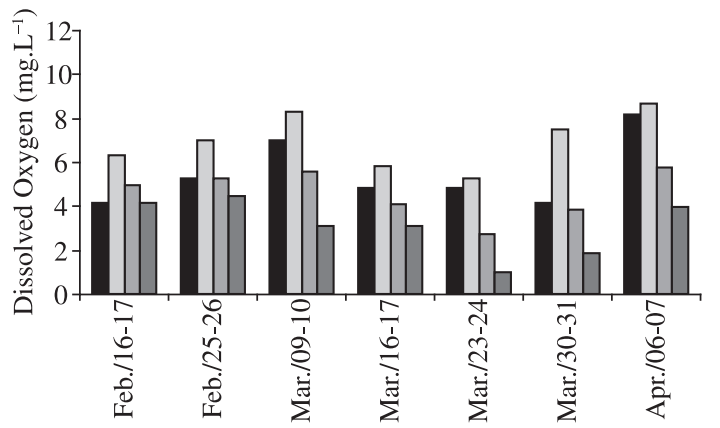

• 12:00 PM 6:00 PM ㅁ12:00 AM 6:00 AM

Figure 2. Diurnal variation of dissolved oxygen in pond 2, in Pindamonhangaba Aquaculture Station, from February to April 1998. oxygen. The water temperature in both ponds varied from 24.5 to $30.8{ }^{\circ} \mathrm{C}$. Water transparency was low during the whole study with values between 0.2 and $0.7 \mathrm{~m}$. The $\mathrm{pH}$ in the ponds varied from slightly acid (6.7) to alkaline (7.7). Water conductivity varied from 42 to $74 \mu \mathrm{S} . \mathrm{cm}^{-1}$ in pond number 1 and from 41 to $70 \mu \mathrm{S} . \mathrm{cm}^{-1}$ in pond number 2. Oxygen concentrations in the water varied between 1.56 and 9.66 in pond number 1 and between 0.97 and 8.65 in pond number 2 , during the diurnal cycle, reaching the minimum at 06:00 AM. The mean value of suspended solids was $16.3 \pm 3.7$, indicating high amounts of particles in the water.

The weekly average dissolved oxygen concentrations varied from 3.4 to $7.0 \mathrm{mg}$. $\mathrm{L}^{-1}$ over the study period. The ammonia concentrations varied from 0.14 to $1.36 \mathrm{mg} . \mathrm{L}^{-1}$, nitrite from 2.1 to $82.9 \mu \mathrm{g} . \mathrm{L}^{-1}$, nitrate from

Table 1. Mean values of physical, chemical and biological variables measured in Fish Pond 1, in Pindamonhangaba Aquaculture Station, during the period between February $16^{\text {th }}$ and April $6^{\text {th }}, 1998$.

\begin{tabular}{|c|c|c|c|c|c|c|c|c|c|c|c|c|c|c|}
\hline & 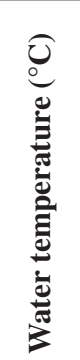 & 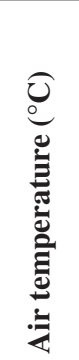 & 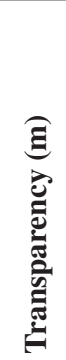 & 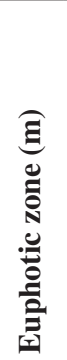 & 졸 & 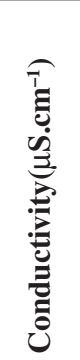 & 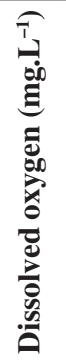 & 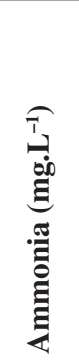 & 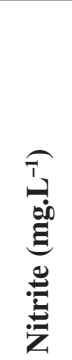 & 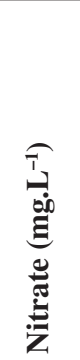 & 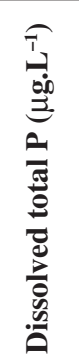 & 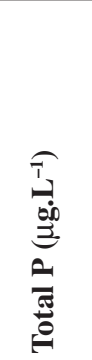 & 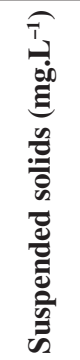 & 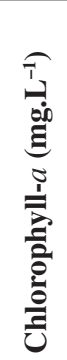 \\
\hline 02/16/1998 & 25.5 & 24.5 & 0.5 & 1.4 & 6.7 & 44.8 & 6.8 & 0.15 & 2.5 & 0.11 & 38.1 & 116.5 & 10.8 & 8.0 \\
\hline 02/25/1998 & 28.5 & 27.5 & 0.6 & 1.6 & 7.4 & 68.0 & 5.8 & 0.31 & 5.0 & 0.12 & 50.4 & 123.9 & 10.4 & 8.2 \\
\hline 03/03/1998 & 29.5 & 29.0 & 0.7 & 1.9 & 7.5 & 73.5 & 5.9 & 0.47 & 4.7 & 0.13 & 53.0 & 81.4 & - & - \\
\hline 03/09/1998 & 30.8 & 29.0 & 0.6 & 1.6 & 7.1 & 42.8 & 5.5 & 0.30 & 3.2 & 0.09 & 32.0 & 113.4 & 21.6 & 22.0 \\
\hline 03/16/1998 & 27.5 & 27.1 & 0.5 & 1.4 & 7.0 & 42.0 & 5.0 & 0.48 & 6.5 & 0.11 & 32.9 & 117.1 & 16.2 & 14.0 \\
\hline 03/23/1998 & 27.5 & 27.5 & 0.5 & 1.4 & 7.2 & 54.0 & 5.1 & 0.54 & 2.8 & 0.11 & 34.3 & 152.9 & 18.8 & 10.6 \\
\hline 03/30/1998 & 27.0 & 27.0 & 0.5 & 1.4 & 6.9 & 41.8 & 5.7 & 1.36 & 82.9 & 0.12 & 52.7 & 219.0 & 29.6 & 17.2 \\
\hline 04/06/1998 & 24.5 & 21.0 & 0.2 & 0.5 & 7.7 & 42.5 & 5.6 & 0.80 & 7.4 & 0.11 & 44.6 & 111.1 & 34.4 & 20.0 \\
\hline
\end{tabular}

Table 2. Mean values of physical, chemical and biological variables measured in Fish Pond 2, in Pindamonhangaba Aquaculture Station, during the period between February $16^{\text {th }}$ and April $6^{\text {th }}, 1998$.

\begin{tabular}{|c|c|c|c|c|c|c|c|c|c|c|c|c|c|c|}
\hline & 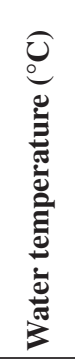 & 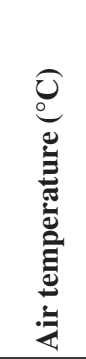 & 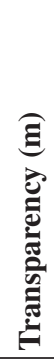 & 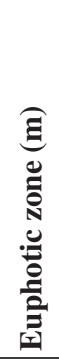 & 플 & 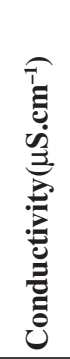 & 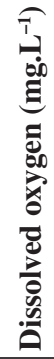 & 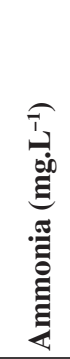 & 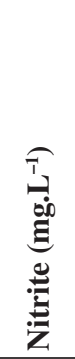 & 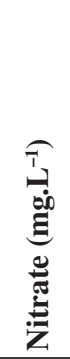 & 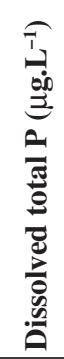 & 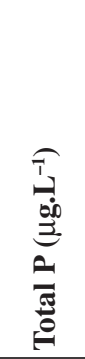 & 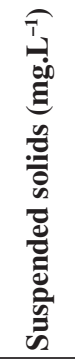 & 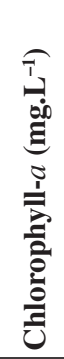 \\
\hline $02 / 16 / 1998$ & 25.5 & 24.5 & 0.5 & 1.4 & 6.8 & 46.5 & 7.0 & 0.14 & 2.8 & 0.14 & 34.2 & 103.9 & 8.0 & 20.9 \\
\hline $02 / 25 / 1998$ & 28.5 & 27.5 & 0.5 & 1.4 & 7.3 & 67.3 & 4.9 & 1.10 & 6.5 & 0.11 & 59.9 & 118.5 & 8.2 & 18.0 \\
\hline 03/03/1998 & 29.5 & 29.0 & 0.7 & 1.9 & 7.3 & 69.9 & 5.5 & 0.43 & 3.9 & 0.13 & 53.0 & 91.8 & - & - \\
\hline 03/09/1998 & 30.8 & 29.0 & 0.7 & 1.9 & 7.1 & 45.3 & 6.0 & 0.22 & 2.3 & 0.08 & 25.0 & 127.9 & 22.0 & 15.1 \\
\hline 03/16/1998 & 27.5 & 27.05 & 0.5 & 1.4 & 6.9 & 42.3 & 4.5 & 0.44 & 4.3 & 0.11 & 26.2 & 105.9 & 14.0 & 26.7 \\
\hline 03/23/1998 & 27.5 & 27.5 & 0.6 & 1.6 & 7.0 & 59.7 & 3.4 & 1.12 & 4.9 & 0.11 & 68.3 & 184.4 & 10.6 & 22.7 \\
\hline 03/30/1998 & 27.0 & 27.0 & 0.4 & 1.0 & 6.7 & 40.8 & 4.3 & 1.18 & 57.4 & 0.09 & 40.1 & 194.2 & 17.2 & 23.4 \\
\hline 04/06/1998 & 24.5 & 21.0 & 0.6 & 1.6 & 7.3 & 43.3 & 6.6 & 0.76 & 2.1 & 0.08 & 27.6 & 96.4 & 20.0 & 48.7 \\
\hline
\end{tabular}


0.08 to $0.14 \mathrm{mg} . \mathrm{L}^{-1}$, total dissolved phosphate from 25.0 to $68.3 \mu \mathrm{g} . \mathrm{L}^{-1}$ and total phosphorus from 81.4 to $219.0 \mu \mathrm{g} . \mathrm{L}^{-1}$.

The zooplankton community was represented by 65 taxa: 14 cladocerans, three copepods and 48 rotifers (Tables 3 and 4, and Figures 3 and 4). A greater richness of species was observed for rotifers, followed by the cladocerans. The richness varied between ponds, the first one having greater richness at the beginning of the study while, in the second pond, richness was greater during the middle of the period of observations. There was a simi- lar tendency in both ponds of a greater richness during the first week, decreasing for the next two weeks, with the lowest value on the $3^{\text {rd }}$ of March, and rising again on the $9^{\text {th }}$. Constant species (with more than $50 \%$ frequency of occurrence in the samples) amongst the cladocerans were Bosmina tubicen, Diaphanosoma spinulosum and Moina minuta; Thermocylops decipiens amongst the Copepods and Asplanchna sieboldi, Asplanchnopus sp., Brachionus calyciflorus, Brachionus caudatus, Conochilus sp., Keratella sp., Hexarthra intermedia and Sinantherina $\mathrm{sp}$.

Table 3. Composition and density (org. $\mathrm{m}^{-3}$ ) of Cladocera and Copepoda in the two Fish Ponds, in Pindamonhangaba Aquaculture Station, from February to April 1998.

\begin{tabular}{|c|c|c|c|c|c|c|c|c|}
\hline & \multicolumn{8}{|c|}{$\mathbf{P 1}$} \\
\hline & $2 / 16 / 98$ & $2 / 25 / 98$ & $3 / 3 / 98$ & $3 / 9 / 98$ & $3 / 16 / 98$ & $3 / 25 / 98$ & $3 / 30 / 98$ & $4 / 6 / 98$ \\
\hline \multicolumn{9}{|l|}{ Cladocera } \\
\hline Bosmina hagmanni (Stingelin, 1904) & 2 & - & - & - & - & - & - & - \\
\hline $\begin{array}{l}\text { Bosmina freyi } \\
\text { (De Melo and Hebert, 1994) }\end{array}$ & 11 & - & - & 23 & - & - & - & - \\
\hline Bosmina tubicen (Brehm, 1953) & 91 & 12 & - & 23 & 34 & 126 & 48 & - \\
\hline $\begin{array}{l}\text { Ceriodaphnia cornuta cornuta } \\
\text { (Sars, 1886) }\end{array}$ & 68 & - & - & 3 & 2 & 25 & 48 & - \\
\hline $\begin{array}{l}\text { Ceriodaphnia cornutaintermedia } \\
\text { (Sars, 1886) }\end{array}$ & 6 & 12 & - & - & 2 & 25 & 5 & 3 \\
\hline $\begin{array}{l}\text { Ceriodaphnia cornuta righaudi } \\
\text { (Sars, 1886) }\end{array}$ & - & - & - & - & - & - & - & 23 \\
\hline Ceriodapnia silvestrii (Daday, 1902) & 46 & 25 & - & - & - & - & - & 5 \\
\hline Daphnia gessneri (Herbst, 1967) & 11 & - & - & - & - & - & - & - \\
\hline Diaphanosoma brevireme (Sars, 1901) & 23 & - & - & - & 23 & 25 & - & 23 \\
\hline $\begin{array}{l}\text { Diaphanosoma spinulosum } \\
\text { (Herbst, 1967) }\end{array}$ & 23 & 25 & 47 & 117 & - & - & 24 & - \\
\hline Diaphanosoma sp. & - & 25 & - & - & - & - & - & - \\
\hline Ilyocryptus spinifer (Herrick, 1882) & - & - & - & - & - & 76 & - & - \\
\hline Macrothrix sp. & - & - & - & 6 & - & - & - & - \\
\hline Moina minuta (Hansen, 1899) & 148 & 147 & 71 & 329 & 928 & 7231 & 5365 & 1664 \\
\hline $\begin{array}{l}\text { Simocephalus latirostris } \\
\text { (Stingelin, 1906) }\end{array}$ & - & - & - & 3 & 928 & - & - & - \\
\hline Simocephalus serrulatus (Koch, 1841) & - & - & - & - & - & - & - & 1 \\
\hline \multicolumn{9}{|l|}{ Copepoda } \\
\hline Adult Calanoida & - & - & - & - & 68 & - & 120 & - \\
\hline Copepodids Calanoida & 297 & 25 & 118 & - & 11 & - & 0 & - \\
\hline Nauplii Calanoida & 365 & 98 & 283 & - & - & - & 120 & 116 \\
\hline Mesocyclops sp. & - & - & - & - & 68 & - & 120 & - \\
\hline Thermocylops decipiens (Kiefer, 1929) & 23 & 74 & - & 23 & - & 101 & 241 & 1040 \\
\hline Copepodids Cyclopoida & 1849 & 2453 & 1321 & 305 & 408 & 2351 & 962 & 3120 \\
\hline \multirow[t]{3}{*}{ Nauplii Cyclopoida } & 1918 & 2208 & 3396 & 2678 & 158 & 379 & 5894 & 11903 \\
\hline & \multicolumn{8}{|c|}{$\mathbf{P 2}$} \\
\hline & 2/16/98 & $2 / 25 / 98$ & $3 / 3 / 98$ & $3 / 9 / 98$ & $3 / 16 / 98$ & $3 / 25 / 98$ & 3/30/98 & $4 / 6 / 98$ \\
\hline \multicolumn{9}{|l|}{ Cladocera } \\
\hline Bosmina hagmanni (Stingelin, 1904) & - & - & - & 12 & - & - & - & - \\
\hline $\begin{array}{l}\text { Bosmina freyi } \\
\text { (De Melo and Hebert, 1994) }\end{array}$ & 12 & - & - & - & 11 & - & - & - \\
\hline Bosmina tubicen (Brehm, 1953) & 25 & 22 & - & 3 & 102 & 146 & 46 & 24 \\
\hline
\end{tabular}


Table 3. Continued...

\begin{tabular}{|c|c|c|c|c|c|c|c|c|}
\hline & \multicolumn{8}{|c|}{$\mathbf{P 2}$} \\
\hline & 2/16/98 & $2 / 25 / 98$ & $3 / 3 / 98$ & $3 / 9 / 98$ & 3/16/98 & $3 / 25 / 98$ & 3/30/98 & $4 / 6 / 98$ \\
\hline $\begin{array}{l}\text { Ceriodaphnia cornuta cornuta } \\
\text { (Sars, 1886) }\end{array}$ & 25 & 22 & - & - & 34 & - & - & 16 \\
\hline Ceriodapnia silvestrii (Daday, 1902) & 25 & 22 & - & 24 & - & - & - & - \\
\hline Daphnia gessneri (Herbst, 1967) & 12 & - & - & - & - & - & - & - \\
\hline Diaphanosoma brevireme (Sars, 1901) & - & - & - & 24 & 136 & 364 & - & - \\
\hline $\begin{array}{l}\text { Diaphanosoma spinulosum } \\
\text { (Herbst, 1967) }\end{array}$ & 12 & - & 25 & 120 & - & - & 69 & 24 \\
\hline Diaphanosoma sp. & - & 86 & - & 12 & - & - & - & - \\
\hline Ilyocryptus spinifer (Herrick, 1882) & - & - & - & 24 & 23 & 36 & 46 & 120 \\
\hline Moina minuta (Hansen, 1899) & 12 & 259 & 50 & 168 & 996 & 4333 & 4923 & 625 \\
\hline $\begin{array}{l}\text { Simocephalus latirostris } \\
\text { (Stingelin, 1906) }\end{array}$ & - & - & - & - & 11 & 36 & - & - \\
\hline \multicolumn{9}{|l|}{ Copepoda } \\
\hline Adult Calanoida & - & - & - & - & - & - & 92 & 241 \\
\hline Copepodids Calanoida & 270 & 130 & 100 & - & - & - & 462 & - \\
\hline Nauplii Calanoida & 589 & 151 & 400 & 24 & - & 73 & 347 & 120 \\
\hline Mesocyclops sp. & - & - & - & - & - & - & 92 & 241 \\
\hline Thermocylops decipiens (Kiefer, 1929) & 12 & 151 & - & - & 11 & 109 & 462 & 722 \\
\hline Copepodids Cyclopoida & 883 & 2463 & 925 & 479 & 1630 & 2003 & 2196 & 5894 \\
\hline Nauplii Cyclopoida & 2723 & 2895 & 4250 & 2875 & 475 & 983 & 6703 & 8540 \\
\hline
\end{tabular}

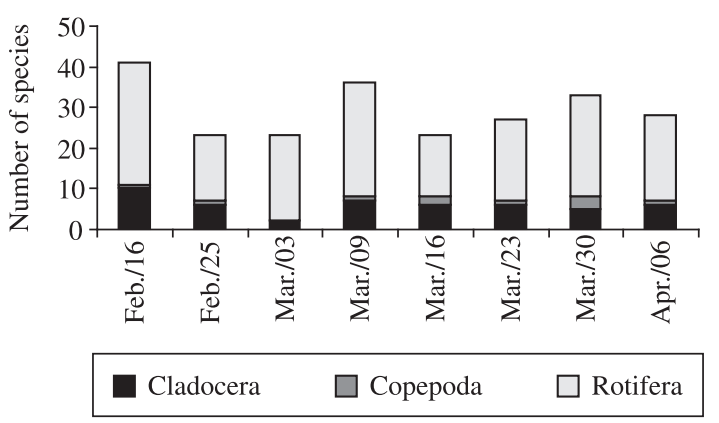

Figure 3. Total species number of the zooplankton communities in pond 1, in Pindamonhangaba Aquaculture Station, from February to April 1998.

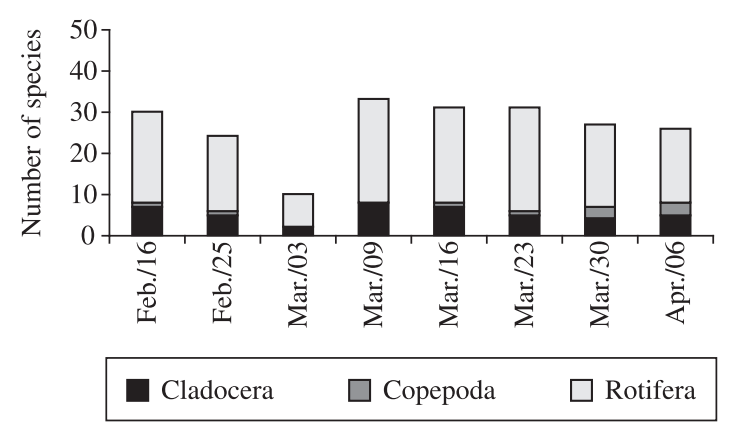

Figure 4. Total species number of the zooplankton communities in pond 2, in Pindamonhangaba Aquaculture Station, from February to April 1998.
In the first weeks of the study, when nutrient quantities were lower, the favoring of smaller organisms like nauplii and copepodids occurred. At the start of the fifth week, along with the increase of nutrients and chlorophyll a, these organisms were substituted by larger forms, like the species Moina minuta, but once again, during the last week of the experiment, another substitution occurred, large forms to smaller forms.

The density of the different species varied between ponds during the entire study period (Tables 3 and 4; Figures 5 and 6). Greatest density occurred in pond 1 on the $6^{\text {th }}$ of April, and in pond 2 on the $23^{\text {rd }}$ of March. Rotifera species reached the highest densities during almost the entire study, especially in pond 1 . In the latter pond, Moina minuta was the most abundant cladocerans, with an increase in density starting in the fourth week. In the second pond, this species was also abundant, but increasing in the fifth week.

In pond 1, dominance of the species Brachionus calyciflorus, B. caudatus and Hexartha intermedia was observed, with high densities during the whole period. In the second pond, Brachionus calyciflorus was found in greatest density, followed by Sinantherina sp. There was a bigger variability in density in pond 2 , with frequent alterations in species dominance throughout the study.

Values of the Shannon Wiener diversity index varied from 0.7 to 1.3 , following the same tendency in both ponds (Figure 7). There was a decrease in the index from the beginning until the third week, rising again afterwards. The smallest index values seen in both ponds coincided with the smallest richness values and with the greatest abundance of Brachionus calyciflorus in pond 1 
Table 4. Composition and density (org. $\mathrm{m}^{-3}$ ) of Rotifera in the two Fish Ponds, in Pindamonhangaba Aquaculture Station, from February to April 1998.

\begin{tabular}{|c|c|c|c|c|c|c|c|c|}
\hline & \multicolumn{8}{|c|}{ P1 } \\
\hline & $2 / 16 / 98$ & $2 / 25 / 98$ & $3 / 3 / 98$ & $3 / 9 / 98$ & $3 / 16 / 98$ & $3 / 25 / 98$ & $3 / 30 / 98$ & $4 / 6 / 98$ \\
\hline \multicolumn{9}{|l|}{ Rotifera } \\
\hline Anuraeopsis sp. & - & - & - & 117 & 340 & - & - & - \\
\hline Ascomorpha sp. & 228 & - & - & 235 & & - & - & 347 \\
\hline $\begin{array}{l}\text { Asplanchna sieboldi } \\
\text { (Leydig, 1854) }\end{array}$ & 114 & - & 1533 & 117 & 1245 & 379 & 241 & - \\
\hline Asplanchnopus sp. & 114 & 491 & 2712 & 1879 & 1925 & 1517 & - & - \\
\hline $\begin{array}{l}\text { Brachionus angularis } \\
\text { (Gosse, } 1851)\end{array}$ & - & - & - & 470 & - & - & 1083 & 1040 \\
\hline $\begin{array}{l}\text { Brachionus calyciflorus } \\
\text { (Pallas, 1766) }\end{array}$ & 685 & 123 & 25708 & 9749 & 3396 & 10745 & 1203 & 693 \\
\hline $\begin{array}{l}\text { Brachionus caudatus } \\
\text { (Barrois and Daday, 1894) }\end{array}$ & 1598 & 3434 & 708 & 4346 & 3396 & 12009 & 4932 & 21611 \\
\hline $\begin{array}{l}\text { Brachionus falcatus } \\
\text { (Zacharias, 1898) }\end{array}$ & 114 & - & - & 117 & - & - & - & - \\
\hline Brachionus mirus (Daday, 1905) & 342 & - & - & - & - & - & - & - \\
\hline Brachionus sp. & 228 & - & 472 & 470 & - & - & - & - \\
\hline $\begin{array}{l}\text { Conochilus coenobasis } \\
\text { (Skorikov, 1914) }\end{array}$ & - & 123 & & 117 & - & 126 & - & 116 \\
\hline Conochilus natans (Seligo, 1990) & 114 & - & - & - & - & 253 & - & - \\
\hline $\begin{array}{l}\text { Conochilus unicornis } \\
\text { (Rousselet, 1892) }\end{array}$ & 1027 & - & - & 352 & - & 379 & 361 & 347 \\
\hline Conochilus sp. & 114 & - & 236 & 1057 & 340 & 1896 & 361 & 116 \\
\hline Dicranophorus sp. & - & - & - & 1292 & - & - & 241 & - \\
\hline $\begin{array}{l}\text { Euchlanis dilatata } \\
\text { (Ehrenber, 1832) }\end{array}$ & - & - & - & - & - & - & - & 116 \\
\hline Euchlanis sp. & 114 & - & - & - & - & 126 & 120 & 116 \\
\hline $\begin{array}{l}\text { Filinia opoliensis } \\
\text { (Zacharias, 1898) }\end{array}$ & - & - & - & & - & - & 120 & \\
\hline Gastropus sp. & - & 736 & - & 705 & - & - & 361 & 116 \\
\hline $\begin{array}{l}\text { Hexartra intermedia } \\
\text { (Wiszniewski, 1929) }\end{array}$ & 1370 & 368 & 1887 & 5050 & 453 & 758 & 120 & 231 \\
\hline $\begin{array}{l}\text { Horaëlla thomassoni } \\
\text { (Koste, 1973) }\end{array}$ & - & - & - & 117 & 113 & - & - & 116 \\
\hline $\begin{array}{l}\text { Keratella americana } \\
\text { (Carlin, 1943) }\end{array}$ & 1826 & 3925 & 1887 & - & - & 126 & 120 & - \\
\hline $\begin{array}{l}\text { Keratella cochlearis } \\
\text { (Gosse, 1851) }\end{array}$ & - & 613 & 354 & 117 & - & 126 & 120 & - \\
\hline Keratella tropica (Apstein, 1907) & - & - & - & - & 1019 & - & - & 347 \\
\hline Keratella sp. & 2968 & 736 & 590 & 940 & - & 379 & - & 347 \\
\hline Lecane bulla (Gosse, 1886) & 114 & - & - & - & - & - & - & - \\
\hline Lecane luna (Müller, 1776) & 114 & - & - & 117 & - & - & 120 & - \\
\hline Lecane lunaris (Ehrenberg, 1832) & - & 123 & 118 & - & - & - & 120 & - \\
\hline Lecane papuana (Murray, 1913) & 342 & - & 118 & - & - & 506 & 1564 & 116 \\
\hline Lecane sp. 1 & 457 & 123 & 118 & - & 113 & & 120 & \\
\hline Lecane sp. 2 & 114 & - & 472 & - & - & - & - & - \\
\hline Lepadella sp. & 1712 & - & 118 & - & - & - & - & - \\
\hline Macrochaetus sp. & - & - & - & 470 & - & - & - & - \\
\hline $\begin{array}{l}\text { Plationus macracanthus } \\
\text { (Daday, 1905) }\end{array}$ & 114 & 123 & - & 1644 & 340 & - & 5 & - \\
\hline Plationus patulus (Müller, 1786) & 46 & - & 354 & 23 & - & - & - & - \\
\hline $\begin{array}{l}\text { Platyias quadricornis } \\
\text { (Ehrenberg, 1832) }\end{array}$ & 114 & - & 118 & - & - & 253 & 120 & - \\
\hline Polyarthra sp. & 114 & 368 & 118 & 470 & 113 & 758 & 361 & 1156 \\
\hline Sinantherina sp. & 114 & 123 & 118 & 587 & - & 2655 & - & 809 \\
\hline Synchaeta sp. & 114 & - & 118 & 235 & 113 & - & 120 & 116 \\
\hline $\begin{array}{l}\text { Testudinella patina } \\
\text { (Hermann, 1783) }\end{array}$ & 114 & 123 & - & 470 & 453 & 126 & 962 & 693 \\
\hline Trichocerca sp. & - & 1840 & 118 & 352 & 113 & - & 120 & - \\
\hline Bdelloidea & 114 & - & - & - & - & 632 & 962 & 578 \\
\hline
\end{tabular}


Table 4. Continued...

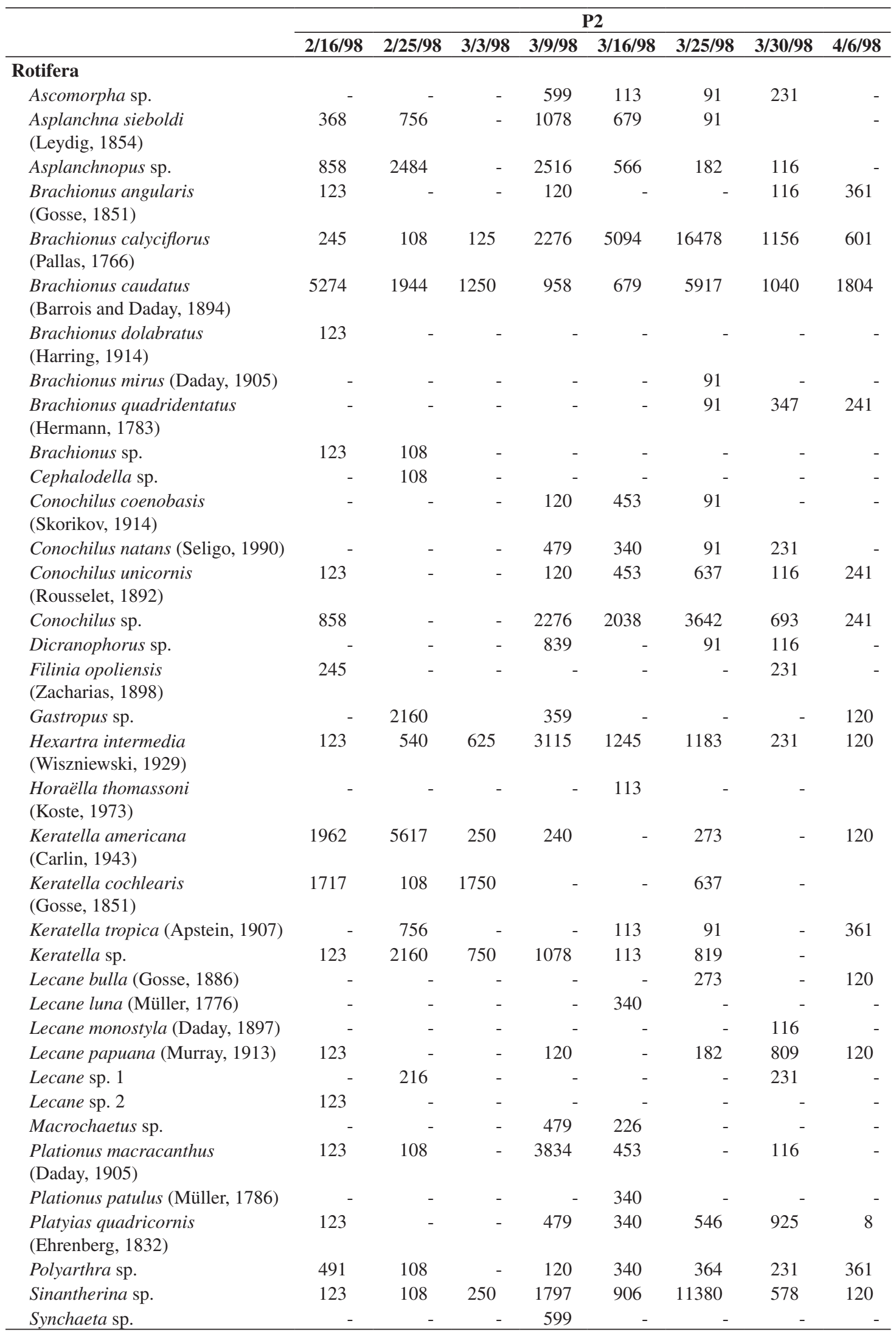


Table 4. Continued...

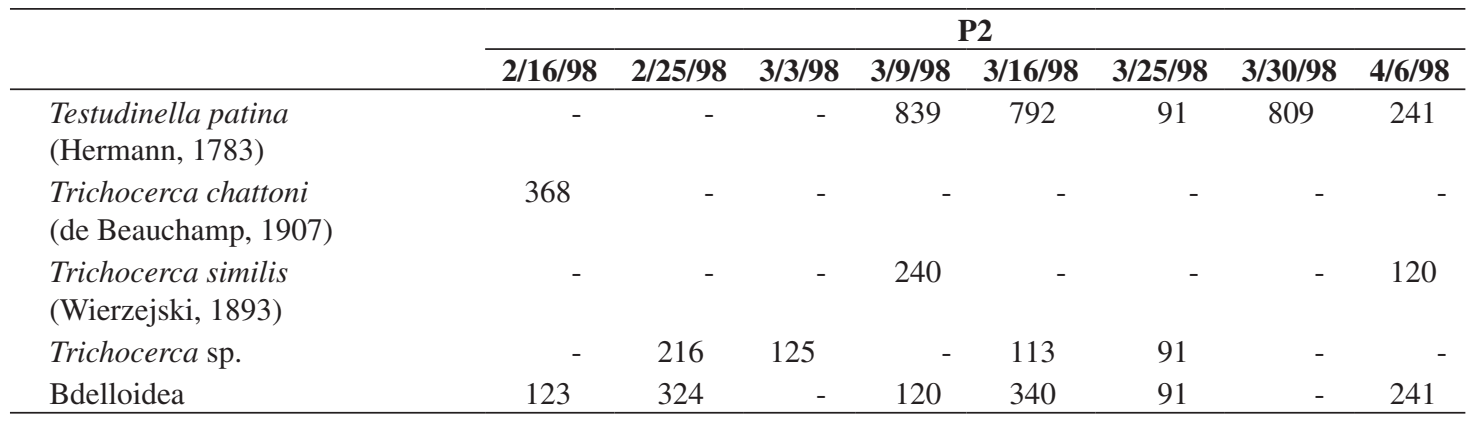

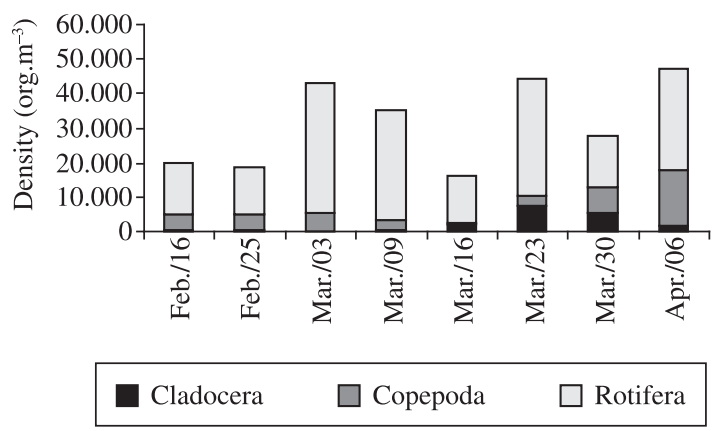

Figure 5. Densities of main zooplankton groups in pond 1, in Pindamonhangaba Aquaculture Station, from February to April 1998.

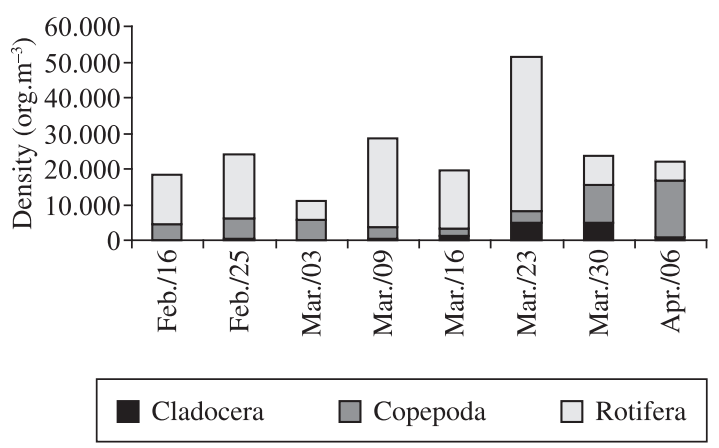

Figure 6. Densities of main zooplankton groups in pond 2, in Pindamonhangaba Aquaculture Station, from February to April 1998.

$\left(25,708 \mathrm{ind} / \mathrm{m}^{3}\right)$ and the third greatest abundance of cyclopoid nauplii in pond $2\left(4,250 \mathrm{ind} / \mathrm{m}^{3}\right)$.

\section{Discussion}

The study was carried out at the end of summer, thus explaining the relatively high water temperatures. Regarding the physical and chemical conditions of the water, it could be observed that the ponds' conditions were adequate for the development of zooplankton, considering that they were relatively well oxygenated, with a slight $\mathrm{pH}$ variation from acid to alkaline, and with a mod-

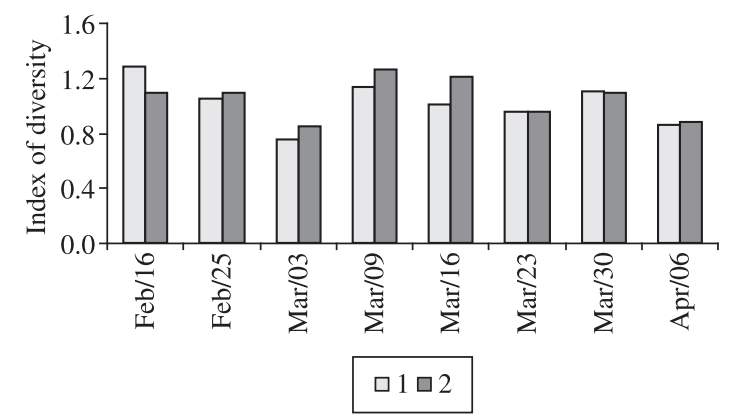

Figure 7. Values of the Shannon-Wiener diversity index for zooplankton communities of fish pond 1 and 2, in Pindamonhangaba Aquaculture Station, from February to April 1998.

erate water conductivity. As evidenced by the inverse relation between water transparency and the concentration of suspended material, the low water transparency was probably partly due to the shallow environment, a fact that favors re-suspension of the sediment, and partly due to the elevated phytoplankton biomass.

According to Sipaúba-Tavares et al. (1994) the conductivity values in eutrophic reservoirs in the Brazilian southeast have been found to reach $240 \mu \mathrm{S} . \mathrm{cm}^{-1}$, but due to the short residence time of the water and the lower accumulation of ions in fish ponds, registered values for the latter environments are generally much lower. Dissolved oxygen concentrations varied greatly during the day, being usually low during the night because of the total community respiration, but high during the day, especially in the afternoon, with oversaturation, caused by photosynthesis (Melo et al., 1988).

Values of ammonia concentrations in the ponds were smaller during the early weeks of the study. Later, the excretion of nitrogen compounds, especially by the Tilapia, could have contributed significantly to the increase of this ion until the end of the study.

The zooplankton community of the fish ponds was characterised by elevated richness of species, as usually observed for this kind of system. Sixty five species were registered in this study, being superior to the value of 59 reported by Eler et al. (2006) for a fishpond in the hydrographic basin of the Mogi-Guaçu river and also 
superior to that found by Sipaúba-Tavares and Colus (1997) in fishponds in the campus of the São Paulo State University, in the municipality of Jaboticabal.

The authors cited above registered a smaller richness of Rotifera species than the present study. Some of the observed species in the present study were also registered by these authors: the cladocerans Bosmina hagmanni, Bosmina freyi, Ceriodaphnia cornuta, Diaphanosoma brevireme, Macrothrix sp. and Moina minuta, the copepod Thermocyclops decipiens, and the rotifers Asplanchna sieboldi, Brachionus angularis, Brachionus calyciflorus, Brachionus caudatus, Brachionus falcatus, Brachionus quadridentatus, Keratella americana, Keratella cochlearis, Keratella tropica, Conochilus unicornis and Trichocerca similis.

Most zooplankton species that occurred in great abundance in this study are commonly found in fish ponds, as found by Eler et al. (2006).

Abundance was greatest for the Rotifera group, during almost all of the study period, followed by Copepoda and Cladocera, in that order. The zooplankton communities were characterised by ample density fluctuations in short periods of time. Such a pattern is characteristic of species with short generation times and quick turnover such as the rotifers and small cladocerans (Sommer et al., 1986)

According to Roche and Rocha (2005), the presence of fish usually reduces the abundance of the biggest zooplankton, especially cladocerans, favouring the increase of small sized zooplankton like rotifers. However, filterfeeding fish may lead to an increase of copepods, whose escape mechanism is well developed, a fact that could suppress the rotifers. Larger species (like Daphnia) may be strongly selected as food items by fish.

Oreochromis niloticus was the fish species present in the ponds during the study. This fish is tolerant to environmental variations, resistant to stress and reproduces readily, being widely cultivated in tropical countries (Baccarin, 1999). In its natural habitat, this species usually feeds on plant material (algae, aquatic plants, seeds, etc.) and small animals (crustaceans, insect larvae, worms, mollusks, amphibians, smaller fish, etc.). It has been reported that this species can efficiently feed on plankton (Kubitza and Kubitza, 2000).

Various zooplankton organisms are utilised by fish as a primary source of food, mainly rotifers, nauplii, copepodite and adult copepods such as the copepod Argyrodiaptomus furcatus (Maia-Barbosa and Matsumura-Tundisi, 1984), and cladocerans such as Moina minuta (Lazzaro, 1987). The latter two may be positively selected due to, respectively, slower movement and eye pigmentation. Moina minuta, considered constant in the present study, is turbidity tolerant (Hart, 1987) and for this reason is common in fish ponds. According to Sipaúba-Tavares and Rocha (2003), this cladoceran has high nutritional value and through parthenogenic reproduction reaches high population densities in short periods of time.
Thermocyclops decipiens is a predator and was also abundant during the study, as found by SantosWisniewski and Rocha (2007) in the eutrophic reservoir of Barra Bonita. The presence of this species may be attributed to its high fecundity and longevity, as well as its short generation time, factors that are important for competition, favouring its predominance (Melão et al., 2005). Rocha et al. (2002) also verified constant occurrence and abundant of this species in eutrophic ecosystems. Leitão et al. (2006), in a study on the zooplankton communities in three reservoirs in the metropolitan area of Fortaleza (Ceará, Brazil), noted the abundance of Thermocyclops decipiens to be related to high ammonia concentrations, as observed in the present study.

As found in the present study, zooplankton communities in eutrophic environments are normally composed of micro-zooplankton (rotifers, protozoans, nauplii and copepodites). (Pinto-Coelho et al., 2005). According to Sipaúba-Tavares et al. (1994), rotifers are more abundant but contribute less to the biomass in zooplankton communities.

Smallest values of the Shannon-Wiener diversity index in both ponds were related to lower species richness and to the dominance of Brachionus calyciflorus. The species Brachionus calyciflorus and B. angularis are cosmopolitans, usually occurring in alkaline waters, and are frequent in fish ponds (Eler et al., 2006). According to the latter authors, these species tolerate organic pollution, being found even in oxidation lagoons and activated sludge. Matsumura-Tundisi (1999) stated that Brachionus calyciflorus and Asphanchna sieboldi are indicators of elevated trophic state; both were constant in the present study.

With regard to the fluctuations in composition and density of the zooplankton observed over short periods of time, there was no regular pattern for zooplankton succession, with species apparently being substituted randomly, during the study period. Environmental characteristics are determinant factors for the occurrence of zooplankton species due to the latter's fast response to any changes in the former. According to Ruttner (1975), many rotifer species are present during the entire year in different densities; however, others apparently disappear in certain periods, reappearing later on. This happens principally because phytoplankton succession can occur over very short time periods, promoting changes in availability and adequacy of food, to which the zooplankton can respond rapidly (Sommer et al., 1986).

\section{References}

American Public Health Association - APHA., 1998. Standard methods for the examination of water and wastewater. $20 \mathrm{ed}$. Washington. $1220 \mathrm{p}$.

BACCARIN, AL., 1999. Desempenho produtivo e características morfológicas do aparelho digestório da tilápia do Nilo (Oreochromis niloticus) alimentada com levedura desidratada de álcool. Jaboticabal: UNESP. 62 p. [Dissertação de Mestrado]. 
DAJOZ, R., 1983. Ecologia Geral. Petrópolis: Vozes. 472 p.

EDMONDSON, WT., 1959. Freshwater Biology. 2 ed. New York: John Wiley. $1248 \mathrm{p}$.

ELER, MN., PARESCHI, DC., NOGUEIRA, AM. and MILANI, TJ., 2006. Avaliação dos impactos de pesque-pague: uma análise da atividade na bacia hidrográfica do rio Mogi-Guaçu. São Carlos: Rima. 312 p.

ELMOOR-LOUREIRO, LMA., 1997. Manual de identificação de cladóceros límnicos do Brasil. Brasília: Universa. 155 p.

FEIDEN, A. and HAYASHI, C., 2005. Desenvolvimento de juvenis de Piracanjuba (Brycon orbignyannus) Vallencienes (1849) (Teleostei: Characidae) em tanques experimentais fertilizados com adubação orgânica. Semina, vol. 26, no. 4, p. 591-600.

GOLTERMAN, HL. and CLYMO, RS., 1969. Methods for physical and chemical analysis of freshwaters. London: Internacional Biological Programme. 172 p.

HART, RC., 1987. Population dynamics and production of five Crustacean zooplankters in subtropical Lake Sibaya. Freshwater biology, vol. 18, no. 2, p. 287-318.

KOSTE, W., 1978. Rotatoria die radertiere mitteleuropas, Übeiordnung Monogononta. Berlim: Gebriider Bernträger. 1010 p.

KUBITZA, F. and KUBITZA, LMM., 2000. Qualidade da água, sistemas de cultivo, planejamento da produção, manejo nutricional e alimentar e sanidade. Panorama da Aqüicultura, vol. 10, no. 59, p. 44-53.

LAZZARO, XA., 1987. A review of planktivorous fishes: their evolution, feeding, behaviours, selectivities and impacts. Hydrobiologia, vol. 146, p. 97-167.

LEITAO, AC., FREIRE, RHF., ROCHA, O. and SANTAELLA, ST., 2006. Zooplankton community composition and abundance in two Brazilian semiarid reservoirs. Acta Limnologica Brasiliensia, vol. 18 , no. 4 , p. 451-468.

MACEDO, CF. and SIPAÚBA-TAVARES, LH., 2005. Comunidade planctônica em viveiros de criação de peixes em disposição sequencial. Boletim do Instituto de Pesca, vol. 31, no. 1, p. 21-27.

MAGURRAN, AE., 1998. Ecological diversity and its measurement. Princeton: Princeton University Press. 179 p.

MAIA-BARBOSA, P. and MATSUMURA-TUNDISI, T., 1984. Consumption of zooplanktonic organisms by Astyanax fasciatus Cuvier, 1819 (Osteichthyes, Characidae) in Lobo (Broa) Reservoir, São Carlos, São Paulo, Brazil. Hydrobiologia, vol. 113, p. 177-181.

MARGALEF, R., 1983. Limnologia. Barcelona: Ômega. 1010 p.

MATSUMURA-TUNDISI, T., 1999. Diversidade do zooplâncton em represas do Brasil. In HENRY, R. (Ed.). Ecologia de reservatórios: estrutura, função e aspectos sociais. Botucatu: FUNDIBIO-FAPESP. $800 \mathrm{p}$

MELÃO, MG., ROCHA O. and ROCHE, KF., 2005. Produtividade, biomassa, flutuações algais e interações biológicas da comunidade planctônica e suas implicações na transferência de energia na cadeia alimentar de um reservatório raso e oligotrófico. In ROCHE, KF. and ROCHA, O. Ecologia trófica de peixes com ênfase na planctivoria em ambientes lênticos de água doce no Brasil. São Carlos: Rima. p. $25-80$.

MELO, JSC., NASCIMENTO, VMC. and LUCAS, AFB., 1988. Variação diurna de temperatura e oxigênio dissolvido em viveiros e tanques do CEPTA, por estações climáticas. Acta Limnologica Brasiliensia, vol. 2, p. 249-259.

PINTO-COELHO, RM., GIANI, A., MORAIS JUNIOR, CA., CARVALHO JUNIOR, ER. and BEZERRA-NETO, JF., 2005. The nutritional status of zooplankton in a tropical reservoir: effects of food quality and community structure. Revista Brasileira de Biologia = Brazilian Journal of Biology, vol. 65, no. 2, p. 313-324.
REID, JW., 1985. Chave de identificação para as espécies continentais sul-americanas de vida livre da Ordem Cyclopoida (Crustacea, Copepoda). Revista Brasileira de Zoologia, vol. 9, p. $17-143$

RIETZLER, AC. and ROCHA, O., 2000. Relações tróficas em ecossistema de água doce. In Anais do I Workshop sobre qualidade da água na aquicultura. Pirassununga: MMA. p. 7-16.

ROCHA, O., MATSUMURA-TUNDISI, T. and TUNDISI, JG., 2002. Hotspots of zooplankton diversity in São Paulo State. Verhandlungen der Internationalen Vereinigung fur Theoretische und Angewandte Limnologie, vol. 28, no. 5, p. 12-16.

ROCHE, KF. and ROCHA, O., 2005. Aspectos de predação por peixes e, lagos e represas, com enfoque na planctivoria. In ROCHE, KF. and ROCHA, O. (Org.). Ecologia trófica de peixes com ênfase na planctivoria em ambientes lênticos de água doce no Brasil. 1 ed. São Carlos: Rima. p. 1-24.

RUTTNER, F., 1975. Fundamentals of limnology. Toronto: University Toronto. $307 \mathrm{p}$.

SAMPAIO, EV., ROCHA, O., MATSUMURA-TUNDISI, T. and TUNDISI, JG., 2002. Composition and abundance of zooplankton in the Limnetic zone of seven reservoirs of the Paranema River, Brazil. Revista Brasileira de Biologia = Brazilian Journal of Biology, vol. 62, no. 3, p. 525-545.

SANTOS-WISNIEWSKI, MJ. and ROCHA, O., 2007. Spatial distribuition and secondary production of Copepoda in a tropical reservoir: Barra Bonita, SP. Revista Brasileira de Biologia = Brazilian Journal of Biology, vol. 67, no. 2, p. 223-233.

SEGERS, H., 1995. Rotifera: the Lecanidae (Monogononta) In DUMONT, HJF. (Ed.). Guides to the identification of the microinvertebrates of the continental waters of the world. Netherlands: SPB Academic. vol. 2, $226 \mathrm{p}$

SILVA, WM. and MATSUMURA-TUNDISI, T., 2005. Taxonomy, ecology, and geographical distribution of the species of the genus thermocyclops kiefer, 1927 (copepoda, cyclopoida) in São Paulo State, Brazil, with description of a new species. Revista Brasileira de Biologia = Brazilian Journal of Biology, vol. 65, no. 3, p. 521-531.

SIPAÚBA-TAVARES, LH. and MORENO, SQ., 1994. Variação dos parâmetros limnológicos em um viveiro de piscicultura nos períodos de seca e chuva. Revista UNIMAR, vol. 16, no. 4, p. 229-242.

SIPAÚBA-TAVARES, LH. and COLUS, DSO., 1997. Estrutura da comunidade fitoplanctônica e zooplanctônica em dois viveiros de cultivo semi-intensivo de peixes (Jaboticabal, São Paulo, Brasil). Boletim do Laboratório de Hidrobiologia, vol. 10, p. 51-64.

SIPAÚBA-TAVARES, LH. DURIGAN, JG. and LIGEIRO, SR., 1994. Caracterização de algumas variáveis limnológicas em um viveiro de piscicultura em dois períodos do dia. Revista UNIMAR, vol. 16 , no. 3 , p. $229-242$

SIPAÚBA-TAVARES, LH., 1996. Variação diurna de alguns parâmetros limnológicos em três viveiros de piscicultura submetidos a diferentes tempos de residência. Acta Limnologica Brasiliensia, vol. 8, p. 29-36.

SIPAÚBA-TAVARES, LH. and ROCHA, O., 2003. Produção de plâncton (fitoplâncton e zooplâncton) para alimentação de organismos aquáticos. São Carlos: Rima. 106 p.

SOMMER, U., GLIWICZ, ZM., LAMPERT, W. and DUNCAN, A., 1986. PEG-model of Seasonal Succession of Planktonic Events in Fresh Waters. Archiv für Hydrobiologie, vol. 106, no. 4, p. $433-471$

WETZEL, RG. and LIKENS, GE., 1991. Limnological analyses. 2 ed. USA: WB Saunders Company. 391 p. 